\title{
Is serum brain-derived neurotrophic factor related to craving for or use of alcohol, cocaine, or methamphetamine?
}

This article was published in the following Dove Press journal:

Neuropsychiatric Disease and Treatment

22 June 2011

Number of times this article has been viewed

\section{Craig Hilburn \\ Vicki A Nejtek \\ Wendy A Underwood \\ Meharvan Singh \\ Gauravkumar Patel \\ Pooja Gangwani \\ Michael J Forster}

University of North Texas Health Science Center at Fort Worth, TX, USA
Correspondence: Craig Hilburn University of North Texas Health Science Center at Fort Worth, Department of Pharmacology and Neuroscience, 3500 Camp Bowie Blvd. CBH, Fort Worth, TX 76107, USA

$\mathrm{Tel}+\mathrm{I} 8177352000$

Fax +l 8174570593

Email craighilburn@att.net
Background: Data suggests that brain-derived neurotropic factor (BDNF) plays a neuroadaptive role in addiction. Whether serum BDNF levels are different in alcohol or psychostimulants as a function of craving is unknown. Here, we examined craving and serum BDNF levels in persons with alcohol versus psychostimulant dependence. Our goals were to explore BDNF as an objective biomarker for 1) craving 2) abstinence, and 3) years of chronic substance use.

Methods: An exploratory, cross-sectional study was designed. Men and women between 20-65 years old with alcohol, cocaine, or methamphetamine dependence were eligible. A craving questionnaire was used to measure alcohol, cocaine and methamphetamine cravings. Serum levels of BDNF were measured using enzyme linked immunoassay. Analysis of variance, chi-square, and correlations were performed using a 95\% confidence interval and a significance level of $P<0.05$.

Results: We found a significant difference in the mean craving score among alcohol, cocaine and methamphetamine dependent subjects. There were no significant influences of race, gender, psychiatric disorder or psychotropic medication on serum BDNF levels. We found that among psychostimulant users BDNF levels were significantly higher in men than in women when the number of abstinent days was statistically controlled. Further, a significant correlation between serum BDNF levels and the number of abstinent days since last psychostimulant use was found.

Conclusion: These data suggest that BDNF may be a biomarker of abstinence in psychostimulant dependent subjects and inform clinicians about treatment initiatives. The results are interpreted with caution due to small sample size and lack of a control group.

Keywords: BDNF, alcohol, cocaine, methamphetamine, craving

\section{Introduction}

Addiction is a progressive disease characterized by chronic use often initiated from substance craving and eventual relapse. ${ }^{1}$ Persons with addictions may also use chronically to alleviate withdrawal symptoms. During stages of withdrawal, neurobiological changes occur in dopamine, serotonin, and gamma-aminobutyric acid (GABA)containing neuron systems. ${ }^{2-4}$ Neurotrophic factors are important neurobiological ligands involved in the development of addiction to drugs and alcohol.

Cerebral brain-derived neurotropic factor (BDNF) is an important modifier of neurogenesis, synaptic plasticity, cognitive functioning, and memory. ${ }^{5,6}$ Changes in BDNF expression in serotonergic and dopaminergic neurons have been found in pathological behaviors. ${ }^{7-9}$ BDNF seems to influence neuronal differentiation of long-term potentiation and neurotransmission in those with psychostimulant and alcohol 
dependence. ${ }^{10-12}$ Preclinical research shows that repeated cocaine administration increases BDNF expression in medial prefrontal cortical tissue. ${ }^{13}$ In rodent self administration models, an injection of BDNF into the ventral tegmental area (VTA) following cocaine administration increases drug seeking behaviors and self-administration. ${ }^{14,15}$ In humans, peripheral serum BDNF levels appear to be significantly correlated with early alcohol withdrawal severity. ${ }^{16}$ In addition, preclinical models have demonstrated that peripheral serum BDNF levels are correlated with BDNF centrally, specifically in cortical regions. ${ }^{17}$ Taken together, these data suggest that BDNF plays a strong neuroadaptive role in the development of addiction to drugs or alcohol.

However, it is still unclear as to whether serum BDNF levels may differentiate alcohol and psychostimulant use in a clinical population or serve as a biomarker of substance use or craving. Here, we examined the relationship between substance craving and serum BDNF levels in persons with alcohol or psychostimulant dependence, specifically cocaine or methamphetamine. Our primary goals were to explore BDNF as an objective biomarker for 1) self-reported craving intensity, 2) current substance use defined as the number of abstinent days since last use, and 3) substance use history defined as the number of years of chronic use.

\section{Methods}

\section{Study design}

A prospective proof-of-concept, cross-sectional study was designed to explore serum BDNF as a biomarker for current craving, abstinence, and years of chronic use in alcohol versus psychostimulant dependent subjects. The study was conducted between September 2009 and May 2010 in an outpatient, community-based setting. In accordance with the Declaration of Helsinki, Institutional Review Board approval by the University of North Texas Health Science Center in Fort Worth was obtained. In addition, a Certificate of Confidentiality was issued by the National Institute of Health to protect this vulnerable sample. Participants received a $\$ 50$ gift card after successfully completing all study assessments and procedures.

\section{Participants}

Volunteers were recruited through pamphlets and advertisements placed in local community-based social service agencies providing counseling and addiction treatment. All volunteers had adequate opportunities to ask researchers questions about the study during the consenting process and make an informed decision whether or not to participate. All eligible subjects provided written informed consent to participate in the study and received copies of the consent form and the Certificate of Confidentiality.

\section{Inclusion/exclusion criteria}

Eligible participants were 20-60 years old, of all ethnicities, and met current Axis I Diagnostic and Statistical Manual of Mental Disorders-IV (DSM-IV) diagnosis for alcohol, cocaine, or methamphetamine dependence. Subjects were English-speaking and had at least an 8th grade education. Volunteers were excluded if they had any current co-morbid substance dependence other than alcohol, cocaine or methamphetamine. Other exclusion criteria were those who had a brain injury or stroke, mental retardation, dementia, HIV/ AIDS, or those who tested positive for current alcohol or any drug use as demonstrated by a breathalyzer and a 12-panel urine drug screen (Redwood Toxicology Laboratory, Santa Rosa, California). Subjects were required to abstain from food and exercise for three to four hours prior to study participation.

\section{Clinical assessments}

Once sociodemographic information was recorded, participants were interviewed using the Mini-International Neuropsychiatric Interview (MINI) to confirm diagnoses and age of onset for lifetime mood and substance use disorders. ${ }^{18}$ The MINI is based on Axis I DSM-IV diagnostic criteria and has an acceptable range of reliability for alcohol and drug dependence ( $a=0.60$ to 0.74 ) and for psychiatric disorders $(a=0.45-0.59) .{ }^{18}$ In addition to its reliability for both substance use and psychiatric disorders, the MINI was used for its brevity (15 minutes) which was more feasible for us to use in our community-based research sample than the timeintensive Structured Clinical Interview for DSM-IV.

The 10-item Cocaine Craving Questionnaire-Brief ${ }^{19}$ was modified to measure subjective alcohol, cocaine and methamphetamine cravings. This instrument measures craving as the lack of control of using, the desire, intention, planning, and anticipation of using to quell withdrawal symptoms. ${ }^{19,20}$ This questionnaire is scored using a Likert scale from 1 to 7 with 1 representing 'strongly disagree' and 7 being 'strongly agree'. Scores are additive. The maximum total score indicating severe cravings is 70 while a score of 10 indicates that no craving is present. This craving instrument was used due to its brevity, high internal reliability ( $\alpha=0.90)$ and concurrent validity. ${ }^{19}$ In addition, previous studies using this questionnaire found this instrument highly effective in measuring methamphetamine and alcohol cravings. ${ }^{21,22}$ 


\section{Serum BDNF analysis}

All testing and sample handling procedures were performed using standard bio-safety techniques and disposal requirements as required by the University of North Texas Health Science Center. Blood specimens were collected only from study participants who satisfied eligibility requirements and tested negative for all drugs and alcohol. Those who had negative toxicology results were allowed to give blood and continue participation in the study. Blood samples were taken between the hours of 9:30 am-10:30 am. Subjects were required to abstain from food or drink for a period of 2-3 hours prior to providing blood samples.

Subjects were placed in a comfortable upright resting position while venous blood samples $(30 \mathrm{~mL})$ were drawn from the antecubital vein into Vacutainer ${ }^{\circledR}$ (BD, Franklin Lakes, $\mathrm{NJ})$ tubes using a butterfly syringe. Blood samples were then centrifuged at $1000 \mathrm{rpm}$ for 5 minutes after collection and serum specimens were stored at $-80{ }^{\circ} \mathrm{C}$ until immunoassays were performed. BDNF was measured according to directions of Promega BDNF Emax Immunoassay System Kit (Promega, Madison, WI, USA). The serum samples were analyzed together in duplicate batch runs to assure an acceptable range of intra-assay coefficient of variation $(\sim 7.1 \%)$ was obtained. Serum BDNF levels are stated as the mean average with standard deviations reported in picograms per milliliter.

\section{Statistical analyses}

Sociodemographic and clinical characteristics were determined using descriptive statistics, analysis of variance, and chi-square analyses, as appropriate. Alcohol, cocaine, and methamphetamine group comparisons of BDNF levels were made using analysis of variance. Pearson correlations were used to examine relationships among serum BDNF levels, craving, current days of abstinence, and the number of years of substance use. Covariates included age, gender, race, psychiatric disorder, and psychotropic medication use. All statistical analyses were performed using SPSS version 17. The statistical program we used automatically deleted outliers during the analysis to prevent spurious results. A 95\% confidence interval and a probability alpha of 0.05 were used to determine statistical significance. To correct for multiple comparisons, Tukey post-hoc analyses were conducted on significantly different grouping variables that were relevant to our primary goals.

\section{Results}

\section{Participants}

Volunteers $(n=105)$ with psychostimulant or alcohol dependence were screened for study eligibility. Nine volunteers did not meet inclusion criteria. Ninety-six volunteers met all study criteria and provided written informed consent to participate. Out of those who provided written informed consent, 32 participants were unable to complete all study assessments. Sixty-four participants provided evaluable data. Sociodemographic characteristics are shown in Table 1.

\section{Sociodemographic and substance use variables}

There were no significant differences in gender distribution among the three substance groups. However, there was a significant race difference as no African-American subjects used methamphetamines: $\chi^{2}(6, \mathrm{n}=64)=19.44$; $P=0.003$. Age among the three subject groups significantly differed $\left(F_{(2,61)}=11.83, P=0.001\right)$. The age difference is reflected in the mean years of use among the substance groups $\left(F_{(2,61)}=4.87, P=0.006\right)$. To control for multiple comparisons, Tukey post hoc analyses showed that years of substance use significantly different between alcohol and methamphetamine $(P=0.012)$ and between alcohol and cocaine $(P=0.009)$. A near significant trend was noted in the mean number of abstinent days that differed among alcohol, cocaine, and methamphetamine groups $\left(F_{(2,61)}=4.50\right.$, $P=0.056)$.

\section{Craving}

Table 1 shows a significant difference in the mean craving score among the three substance groups $\left(F_{(2,61)}=4.50\right.$, $P=0.015)$. To be more specific, we used Tukey post hoc comparisons and found a significant difference in craving between the alcohol and cocaine groups $(P=0.012)$. There were no statistical differences in craving between alcohol and methamphetamine $(P=0.756)$ or between cocaine and methamphetamine groups $(P=0.162)$. To examine the general effect of demographic and clinical indices on craving we collapsed the substance groups and analyzed mean craving scores according to race, gender, psychiatric disorder and psychotropic medication groups. Table 2 shows no significant demographic or clinical group differences in craving.

\section{Serum BDNF levels, sociodemographic and clinical indices}

Table 2 shows that there was no significant influence of race, gender, psychiatric disorder or psychotropic medication on serum BDNF levels. There were no significant gender differences in serum BDNF levels between men and women $\left(F_{(1,62)}=3.36, P=0.071\right)$. Serum BDNF levels did not differ significantly among alcohol, cocaine, and methamphetamine 
Table I Sample and substance use characteristics

\begin{tabular}{|c|c|c|c|c|c|}
\hline Demographic variables & Alcohol $(\mathbf{N}=27)$ & Cocaine $(N=22)$ & Methamphetamine $(\mathrm{N}=15)$ & $\chi^{2}$ & $P$ \\
\hline Percentage distributions & $\mathrm{N}(\%)$ & $\mathrm{N}(\%)$ & $\mathrm{N}(\%)$ & & \\
\hline Gender & & & & 0.166 & 0.944 \\
\hline Men & $2 \mathrm{I}(32.8 \mathrm{I})$ & $17(26.56)$ & II (I7.19) & & \\
\hline Women & $6(9.38)$ & $5(7.81)$ & $4(6.25)$ & & \\
\hline Race & & & & 19.44 & 0.003 \\
\hline White & $19(29.69)$ & $7(10.94)$ & $12(18.75)$ & & \\
\hline Black & $6(9.38)$ & $13(20.31)$ & 0 & & \\
\hline Hispanic & $2(3.13)$ & $2(3.13)$ & $2(3.13)$ & & \\
\hline Asian & 0 & 0 & $\mathrm{I}(\mathrm{I} .56)$ & & \\
\hline Substance-related variables & Mean (SD) & Mean (SD) & Mean (SD) & $\boldsymbol{F}$ & $P$ \\
\hline Years of use & $28.667(11.25)$ & $29.409(10.83)$ & $18.33(9.88)$ & 4.87 & 0.006 \\
\hline Number of abstinent days & $70.148(45.94)$ & $108.36(58.104)$ & $76.333(68.856)$ & 3.03 & 0.056 \\
\hline Craving scores & $35.15(16.67)$ & $22.05(13.76)$ & $31.60(15.40)$ & 4.50 & 0.015 \\
\hline Serum BDNF pg/mL & $2853.07(2112.80)$ & $3660.39(2377.80)$ & $3779.39(2803.49)$ & 1.02 & 0.366 \\
\hline \multicolumn{6}{|l|}{ Tukey post hoc comparisons } \\
\hline Substance-related variables & (I) & (J) & Mean difference (I-J) & Std. error & $P$ \\
\hline \multirow[t]{3}{*}{ Years of substance use } & Alcohol & Cocaine & -0.74 & 3.10 & 0.969 \\
\hline & & Methamphetamine & 10.33 & 3.48 & 0.012 \\
\hline & Cocaine & Methamphetamine & 11.08 & 3.62 & 0.009 \\
\hline \multirow[t]{3}{*}{ Craving scores } & Alcohol & Cocaine & 13.10 & 4.43 & 0.01 \\
\hline & & Methamphetamine & 3.55 & 4.97 & 0.76 \\
\hline & Cocaine & Methamphetamine & -9.55 & 5.17 & 0.16 \\
\hline
\end{tabular}

Abbreviation: SD, standard deviation.

groups $\left(F_{(2,61)}=1.02, P=0.366\right)$. We analyzed the influence race, gender, psychiatric disorder or psychotropic medication may have had on serum BDNF levels among the substance groups, but there were no significant differences $(P \geq 0.260)$. The mean serum BDNF level in methamphetamine abusers and the relationship with number of days' abstinence was not significant $(r=-0.503, P=0.056)$. Similarly, BDNF levels in alcohol $(r=-0.300, P=0.128)$ and cocaine $(r=-0.185$, $P=0.409)$ abusers were not significantly related to the number of abstinent days. Thus, we collapsed the cocaine and methamphetamine groups and designated this combined sample as the psychostimulant group.

Table 2 BDNF levels and craving scores

\begin{tabular}{|c|c|c|c|c|}
\hline \multirow[t]{2}{*}{ Sample demographics } & \multicolumn{2}{|l|}{ Serum BDNF } & \multicolumn{2}{|c|}{ Craving scores } \\
\hline & Mean (SD) & $F(P)$ & Mean (SD) & $F(P)$ \\
\hline Gender & & $3.366(0.07 I)$ & & $0.020(0.890)$ \\
\hline Men $(N=49)$ & $3644.12(2456.36)$ & & $29.653(14.94)$ & \\
\hline Women $(N=15)$ & $2379.92(\mid 859.6 I)$ & & $30.333(20.60)$ & \\
\hline Race & & I.45। (0.237) & & $0.637(0.594)$ \\
\hline White $(N=38)$ & $3|49.4|$ (259I.87) & & $31.42(16.86)$ & \\
\hline Black $(\mathrm{N}=19)$ & $3694.33(2043.10)$ & & $25.79(15.74)$ & \\
\hline Hispanic $(\mathrm{N}=6)$ & $2790.89(1310.22)$ & & $33.33(15.36)$ & \\
\hline Asian $(\mathrm{N}=\mathrm{I})$ & $7645.5 \mathrm{I}(0.00)$ & & $24.00(0.00)$ & \\
\hline \multicolumn{5}{|l|}{ Clinical indices } \\
\hline Psychiatric disorder & & $1.217(0.303)$ & & $0.875(0.422)$ \\
\hline None $(\mathrm{N}=29)$ & 3796.11 (2505.80) & & $28.14(14.52)$ & \\
\hline Bipolar disorder $(\mathrm{N}=33)$ & $3048.70(2265.94)$ & & $31.94(17.80)$ & \\
\hline Major depression $(\mathrm{N}=2)$ & I783.22 (I6II.65) & & $19.00(12.73)$ & \\
\hline Psychotropic medications & & I.I65 (0.33I) & & $0.226(0.878)$ \\
\hline None $(\mathrm{N}=40)$ & $37 \mid I .58$ (256।.87) & & $30.38(16.02)$ & \\
\hline Antidepressant $(\mathrm{N}=13)$ & $2297.38(1567.45)$ & & 27.17 (19.27) & \\
\hline Antipsychotic $(\mathrm{N}=9)$ & $2970.02(2395.86)$ & & $33.11(15.74)$ & \\
\hline Mood stabilizer $(\mathrm{N}=2)$ & $3838.19(3477.60)$ & & $30.00(16.97)$ & \\
\hline
\end{tabular}

Abbreviations: BDNF, brain-derived neurotropic factor; SD, standard deviation. 


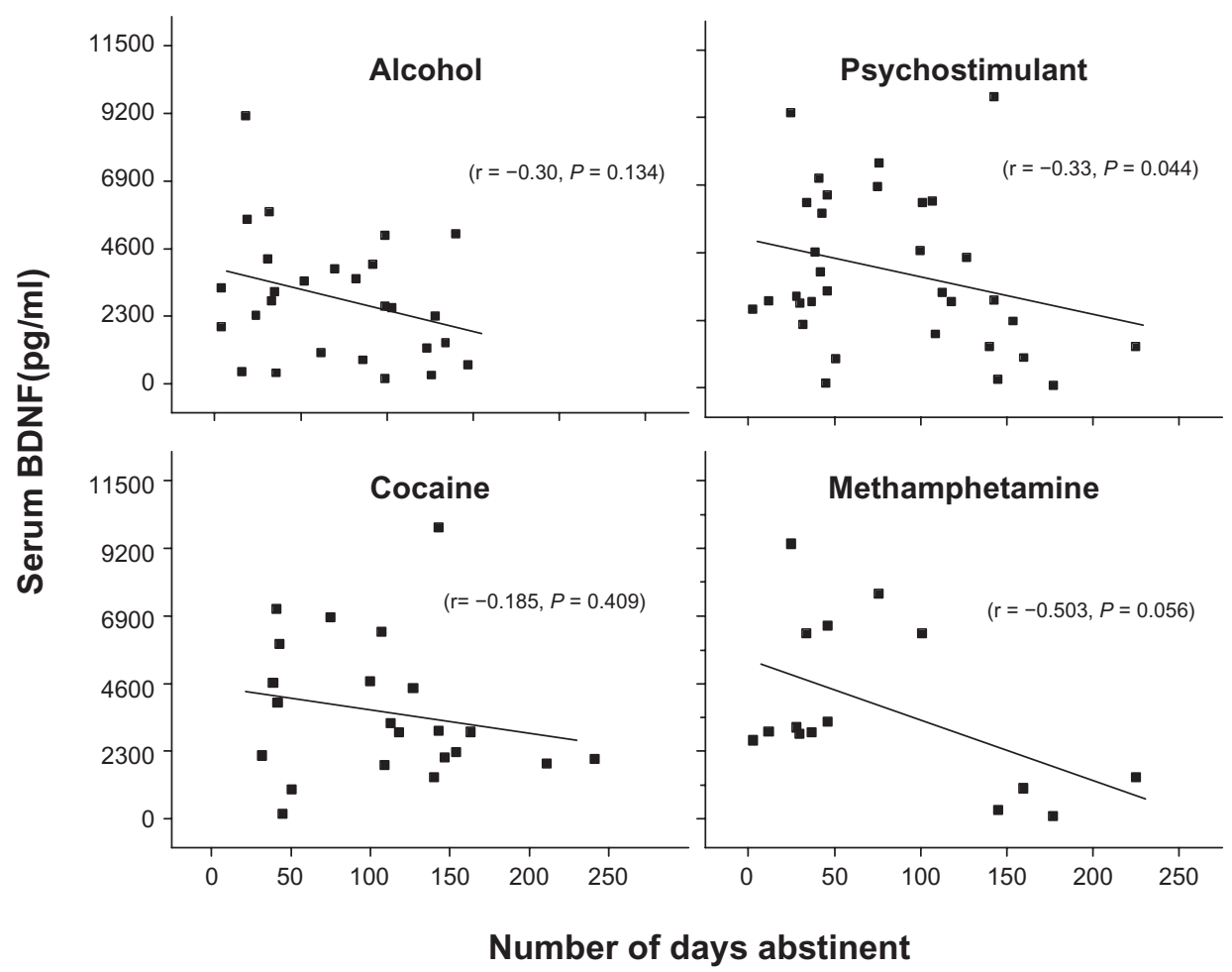

Figure I Correlations between the number of abstinent days and serum brain-derived neurotropic factor levels in alcohol, psychostimulant (cocaine and methamphetamine groups combined), cocaine, and methamphetamine abusers.

\section{Serum BDNF levels in alcohol and psychostimulant groups}

Although serum BDNF levels were lower in the alcohol group in comparison to the psychostimulant group, the difference was not significant $\left(F_{(1,62)}=2.05, P=0.157\right)$. However, a significant difference in serum BDNF levels between alcohol and psychostimulant groups emerged when the number of abstinent days prior to collecting serum BDNF was statistically controlled $\left(F_{(1,61)}=4.31, P=0.045\right)$. In addition, Figure 1 shows a significant correlation between serum BDNF levels and the number of abstinent days in the psychostimulant group $(r=-0.33, P=0.044)$. Serum BDNF levels of alcohol, cocaine, methamphetamine, and the combined cocaine and methamphetamine group (ie, psychostimulant) are shown for visual comparison.

\section{Discussion}

Our research goals were to compare serum BDNF levels in persons with alcohol, cocaine and methamphetamine dependence to craving, abstinence, and years of use. Previous clinical and preclinical BDNF research have focused largely on the serum BDNF levels during periods of withdrawal and post-withdrawal. ${ }^{16,23-26}$ Thus, we may be the first to show differences in BDNF levels among alcohol, cocaine, and methamphetamine use in relation to craving and abstinence. To that end, we found significant differences in BDNF levels between alcohol and psychostimulants as a function of abstinence. However, in terms of substance use history, we found no significant relationships between BDNF and years of chronic use of alcohol, cocaine or methamphetamine. Statistically controlling for the presence of a psychiatric disorder, gender, age, and race did not change these results. Likewise, no significance relationships between BDNF and self-reported craving score for either alcohol, cocaine, or methamphetamine dependent groups emerged. Arguably, the inter- and intra-individual variability of selfreported craving in alcohol and psychostimulant dependent populations may be considerable and transient. Thus, in this cross-sectional design a one-time measure of self-reported craving may not be a suitable variable to correlate with serum BDNF levels.

Many of our subjects had comorbid psychiatric and substance use disorders. There are few studies investigating differences in serum BDNF levels in those with or without comorbid Axis I disorders. One study investigated serum BDNF levels in schizophrenia subjects with and without substance abuse and in healthy controls. ${ }^{23}$ These results showed that serum BDNF levels in schizophrenia patients with co-morbid polysubstance abuse were higher compared to schizophrenia patients who did not abuse substances, 
or healthy controls. Although not significant, we found somewhat lower levels of BDNF levels in those who had comorbid bipolar or major depressive disorders versus those who were not mentally ill. Similarly, de Oliveira et $\mathrm{al}^{24}$ examined serum BDNF levels in bipolar disorder patients who were medication-free, those receiving antipsychotics, antidepressants or mood stabilizers, and healthy controls. ${ }^{24}$ This study reported significantly lower BDNF levels in all bipolar patients regardless of medication use in comparison to healthy controls.

While our study was not designed to examine medication effects per se, we nonetheless examined the effect that psychotropic medications may have had on BDNF levels and craving in those who were receiving psychotropic medications versus those who were medication free. We found BDNF levels were somewhat lower in those taking antidepressant medications although this effect was not statistically different. In contrast to our data, Aydemir et $\mathrm{al}^{25}$ who examined BDNF levels in pre- and 6-weeks post-medication conditions in depressed women $(n=20)$ receiving S-citalopram found a significant increase in BDNF in the post-medication condition.

\section{Study limitations and strengths}

The sample size in the present study may be small in comparison to some clinical trials. However, our sample is substantially larger than Huang et $\mathrm{al}^{16}$ who examined BDNF in persons with alcohol dependence $(\mathrm{n}=47)$, Aydemir et $\mathrm{al}^{25}$ who examined serum BDNF in depressed women $(n=20)$, or Angelluci et $\mathrm{al}^{26}$ who investigated serum BDNF in chronic cocaine and heroin abusers $(n=45)$. The available data is limited to only a handful of small clinical studies and much of the BDNF research is preclinical rodent studies. Thus, our data clearly augments the existing literature.

Another potential limitation is not having controlled for the duration, frequency of use, and average dose of alcohol or the psychostimulants among our subject population. While we acknowledge the importance and interest of these measures the ability to control for these variables would have been beyond the scope of this study. However, we were able to clarify the state of dependence as our subjects qualified for DSM-IV criteria for dependence which covers a 12-month period. Therefore under the rubric of DSM-IV dependence general frequency is implied for 12 months.

Another limitation of our study is the heterogeneity of our subject population. We did not have a healthy control group to compare BDNF levels to the alcohol and psychostimulant groups. We also did not exclude subjects based on comorbities, endocrine disorders or class of psychiatric medication which are known factors that influence BDNF. ${ }^{27-30}$ In doing so, our enrollment goal would have been significantly reduced. Instead, our sample reflects the typical community-based outpatient that clinicians are most likely to encounter. Thus, our study has strong ecological validity producing data that may be helpful to health professionals and case managers.

\section{Concerning therapeutic approaches}

As BDNF comparison studies between alcohol and psychostimulant abusers are scarce, our primary goal was to conduct a proof-of-concept study to generate hypotheses and to investigate differences between alcohol and psychostimulant users. Thus, a control group was not needed. The statistically significant differences we found between alcohol and psychostimulant dependence serve to generate hypotheses rather than to support a particular theory. It would also be of interest to examine the effect of BDNF polymorphism on these data. The distribution of the BDNF-gene 66 ValMet polymorphism results in higher BDNF secretion centrally, and is associated with addiction to drugs and alcohol. ${ }^{31-33}$

There is a possibility that the relationship observed between serum BDNF and number of days abstinent may not be causally related to the induction of the neurobiological substrates which underlie addiction. For example other underlying factors, such as inflammation, could have influenced BDNF in the periphery. ${ }^{34}$ However we excluded subjects with any kind of central nervous system diseases or impairments, as well as HIV/AIDS. Thus the likelihood of brain inflammation is limited. In addition, a number of researchers have suggested that peripheral BDNF concentrations are associated with BDNF levels in the brain..$^{35}$ Indeed, cerebral BDNF crosses the blood-brain barrier, is correlated with BDNF centrally in rodents, and is associated with a marker of neuronal integrity, $\mathrm{N}$-acetylaspartate. ${ }^{17,36,37}$ As this was a proof-of-concept, hypothesis generating study, our data importantly indicate that serum BDNF has feasibility in clinical research of addiction and co-occurring disorders and should be further examined as a biomarker for psychostimulant use and abstinence in future longitudinal research. The results from this study provide applicable substance group differences, thus a larger sample size with added controls in future research with BDNF is substantiated.

\section{Conclusion}

Serum BDNF levels in psychostimulant dependent subjects were significantly related to the number of abstinent days since last psychostimulant use. Psychostimulant dependent 
subjects had significantly higher serum BDNF levels than those with alcohol dependence. Craving and substance use history was not related to serum BDNF levels. These data lend support to the idea that serum BDNF levels may be a biomarker of abstinence, particularly in cocaine and methamphetamine dependence. While these data may inform clinicians, we interpret these results with caution.

\section{Role of funding}

This research was supported by a National Institute of Aging T32 grant AG020494.

\section{Disclosure}

The authors have no conflicts of interest to declare.

\section{Contributions}

Dr Hilburn was the principal investigator in the study, who managed all aspects of the research including clinical design; subject interviewing; data collection; biological and statistical analyses; and manuscript writing. Dr Nejtek assisted Dr Hilburn with clinical design; subject interviewing; data analyses and interpretation; and manuscript writing. Gurav Patel collected and analyzed blood and urine specimens; interviewed subjects; and assisted Dr Hilburn with database management and analyses. Wendy Underwood assisted Dr Hilburn with ELISA biological assays and analyses. Pooja Gangwani collected and analyzed urine and breath samples; interviewed subjects; and assisted Drs Hilburn and Patel with database management. Drs Forster and Singh provided Dr Hilburn biological and technical support concerning BDNF data interpretation and analyses. All authors contributed to and approved the final manuscript.

\section{References}

1. Weiss F. Neurobiology of craving, conditioned reward and relapse. Curr Opin Pharmacol. 2005;5(1):9-19.

2. Cameron DL, Williams JT. Cocaine inhibits GABA release in the VTA through endogenous 5-HT. J Neurosci. 1994;14(11 Pt 1):6763-6767.

3. Lallemant AM, Bralet J. The effect of cocaine treatment on the level of dopamine and serotonin regeneration and on the level of acetylcholine in rat brain. C R Seances Soc Biol Fil. 1976;170(5):1056-1063.

4. Torre E, Bogetto F. Alcohol and neurotransmitters. Review of literature and experimental research. Minerva Psichiatr. 1981;22(1):7-12.

5. Huang EJ, Reichardt LF. Neurotrophins: roles in neuronal development and function. Annu Rev Neurosci. 2001;24:677-736.

6. Lu L, Grimm JW, Shaham Y, Hope BT. Molecular neuroadaptations in the accumbens and ventral tegmental area during the first 90 days of forced abstinence from cocaine self-administration in rats. J Neurochem. 2003;85(6):1604-1613.

7. Berton O, McClung CA, Dileone RJ, et al. Essential role of BDNF in the mesolimbic dopamine pathway in social defeat stress. Science. 2006; 311(5672):864-868.

8. Dluzen DE, Story GM, Xu K, Kucera J, Walro JM. Alterations in nigrostriatal dopaminergic function within BDNF mutant mice. Exp Neurol. 1999;160(2):500-507.
9. Martinowich K, Lu B. Interaction between BDNF and serotonin: role in mood disorders. Neuropsychopharmacology. 2008;33(1):73-83.

10. Matsushita S, Kimura M, Miyakawa T, et al. Association study of brain-derived neurotrophic factor gene polymorphism and alcoholism. Alcohol Clin Exp Res. 2004;28(11):1609-1612.

11. Hyman C, Hofer M, Barde YA, et al. BDNF is a neurotrophic factor for dopaminergic neurons of the substantia nigra. Nature. 1991;350(6315): 230-232.

12. Pang PT, Lu B. Regulation of late-phase LTP and long-term memory in normal and aging hippocampus: role of secreted proteins tPA and BDNF. Ageing Res Rev. 2004;3(4):407-430.

13. Lu B. BDNF and activity-dependent synaptic modulation. Learn Mem. 2003;10(2):86-98.

14. Graham DL, Edwards S, Bachtell RK, DiLeone RJ, Rios M, Self DW. Dynamic BDNF activity in nucleus accumbens with cocaine use increases self-administration and relapse. Nat Neurosci. 2007;10(8):1029-1037.

15. Lu L, Dempsey J, Liu SY, Bossert JM, Shaham Y. A single infusion of brain-derived neurotrophic factor into the ventral tegmental area induces long-lasting potentiation of cocaine seeking after withdrawal. J Neurosci. 2004;24(7):1604-1611.

16. Huang $\mathrm{MC}$, Chen $\mathrm{CH}$, Chen $\mathrm{CH}$, et al. Alterations of serum brainderived neurotrophic factor levels in early alcohol withdrawal. Alcohol. 2008;43(3):241-245.

17. Karege F, Schwald M, Cisse M. Postnatal developmental profile of brain-derived neurotrophic factor in rat brain and platelets. Neurosci Lett. 2002;328(3):261-264.

18. Sheehan DV, Lecrubier Y, Sheehan KH, et al. The Mini-International Neuropsychiatric Interview (M.I.N.I.): the development and validation of a structured diagnostic psychiatric interview for DSM-IV and ICD-10. J Clin Psychiatry. 1998;59 Suppl 20:22-33;quiz 34-57.

19. Sussner BD, Smelson DA, Rodrigues S, Kline A, Losonczy M, Ziedonis D. The validity and reliability of a brief measure of cocaine craving. Drug Alcohol Depend. 2006;83(3):233-237.

20. Tiffany ST, Singleton E, Haertzen CA, Henningfield JE. The development of a cocaine craving questionnaire. Drug Alcohol Depend. 1993; 34(1):19-28.

21. Nejtek VA, Avila M, Chen LA, et al. Do atypical antipsychotics effectively treat co-occurring bipolar disorder and stimulant dependence? A randomized, double-blind trial. J Clin Psychiatry. 2008;69(8): 1257-1266.

22. Brown ES, Nejtek VA, Perantie DC, Rajan Thomas N, Rush AJ. Cocaine and amphetamine use in patients with psychiatric illness: a randomized trial of typical antipsychotic continuation or discontinuation. J Clin Psychopharmacol. 2003;23(4):384-388.

23. Jockers-Scherubl MC, Danker-Hopfe H, Mahlberg R, et al. Brainderived neurotrophic factor serum concentrations are increased in drug-naive schizophrenic patients with chronic cannabis abuse and multiple substance abuse. Neurosci Lett. 2004;371(1):79-83.

24. de Oliveira GS, Cereser KM, Fernandes BS, et al. Decreased brainderived neurotrophic factor in medicated and drug-free bipolar patients. J Psychiatr Res. 2009;43(14):1171-1174.

25. Aydemir C, Yalcin ES, Aksaray S, et al. Brain-derived neurotrophic factor (BDNF) changes in the serum of depressed women. Prog Neuropsychopharmacol Biol Psychiatry. 2006;30(7):1256-1260.

26. Angelucci F, Ricci V, Pomponi M, et al. Chronic heroin and cocaine abuse is associated with decreased serum concentrations of the nerve growth factor and brain-derived neurotrophic factor. J Psychopharmacol. 2007;21(8):820-825.

27. Post RM. Role of BDNF in bipolar and unipolar disorder: clinical and theoretical implications. J Psychiatr Res. 2007;41:979-990.

28. Yoshimura R, Ikenouchi-Sugita A, Hori H, Umene-Nakano W, Katsuki A, Hayashi K, et al. Brain-derived neurotrophic factor (BDNF) and mood disorder. Nihon Shinkei Seishin Yakurigaku Zasshi. 2010;30(5-6):181-184.

29. Hashimoto K. Brain-derived neurotrophic factor as a biomarker for mood disorders: an historical overview and future directions. Psychiatry Clin Neurosci. 2010;64(4):341-357. 
30. Nakagawa T, Tsuchida A, Itakura Y, et al. Brain-derived neurotrophic factor regulates glucose metabolism by modulating energy balance in diabetic mice. Diabetes. 2000;49(3):436-444.

31. Pierce RC, Bari AA. The role of neurotrophic factors in psychostimulant-induced behavioral and neuronal plasticity. Rev Neurosci. 2001;12(2):95-110.

32. Bolanos CA, Nestler EJ. Neurotrophic mechanisms in drug addiction. Neuromolecular Med. 2004;5(1):69-83.

33. Cheng CY, Hong CJ, Yu YW, Chen TJ, Wu HC, Tsai SJ. Brain-derived neurotrophic factor (Val66Met) genetic polymorphism is associated with substance abuse in males. Brain Res Mol Brain Res. 2005; 140(1-2):86-90.

34. Kerschensteiner M, Gallmeier E, Behrens L, et al. Activated human $\mathrm{T}$ cells, B cells, and monocytes produce brain-derived neurotrophic factor in vitro and in inflammatory brain lesions: a neuroprotective role of inflammation? J Exp Med. 1999;189(5):865-870.
35. Gass P, Hellweg R. Peripheral brain-derived neurotrophic factor (BDNF) as a biomarker for affective disorders? Int J Neuropsychopharmacol. 2010;13(1):1-4.

36. Moffett JR, Ross B, Arun P, Madhavarao CN, Namboodiri AM. $\mathrm{N}$-Acetylaspartate in the CNS: from neurodiagnostics to neurobiology. Prog Neurobiol. 2007;81(2):89-131.

37. Pan W, Banks WA, Fasold MB, Bluth J, Kastin AJ. Transport of brain-derived neurotrophic factor across the blood-brain barrier. Neuropharmacology. 1998;37(12):1553-1561.

\section{Publish your work in this journal}

Neuropsychiatric Disease and Treatment is an international, peerreviewed journal of clinical therapeutics and pharmacology focusing on concise rapid reporting of clinical or pre-clinical studies on a range of neuropsychiatric and neurological disorders. This journal is indexed on PubMed Central, the 'PsycINFO' database and CAS, and is the official journal of The International Neuropsychiatric Association (INA). The manuscript management system is completely online and includes a very quick and fair peer-review system, which is all easy to use. Visit http://www.dovepress.com/testimonials.php to read real quotes from published authors.

Submit your manuscript here: http://www.dovepress.com/neuropsychiatric-disease-and-treatment-journal 